\title{
Asymptotic law of limit distribution for fractional Ornstein-Uhlenbeck process
}

Liang Shen ${ }^{1,2}$ and Qingsong X $\mathrm{u}^{1 *}$

"Correspondence:

csuqingsongxu@126.com

1 School of Mathematics and

Statistics, Central South University,

Changsha, China

Full list of author information is

available at the end of the article

\begin{abstract}
We consider the minimum $L_{1}$-norm estimator $\theta_{\varepsilon}^{*}$ of the parameter $\theta$ of a linear stochastic differential equation $d X_{t}=\theta X_{t} d t+\varepsilon d B_{t}^{H}, X_{0}=x_{0}$, where $\left\{B_{t}^{H}, 0 \leq t \leq T\right\}$ is a fractional Brownian motion. The asymptotic law of its limit distribution is studied for $T \rightarrow+\infty$, when $\varepsilon \rightarrow 0$.
\end{abstract}

Keywords: fractional Ornstein-Uhlenbeck process; minimum $L_{1}$-norm estimator; fractional Brownian motion; asymptotic law

\section{Introduction}

Stochastic differential equations driven by Brownian motions are used widely in variety of sciences as stochastic modeling to describe some phenomena. There are many applications such as mathematical finance, economic processes as well as signal processing. The Ornstein-Uhlenbeck process, which is also called the Vasicek model in finance, is being extensively used in finance over the last few decades as the one-factor short-term interest rate model. Statistical inference for the process of Ornstein-Uhlenbeck type driven by Brownian motions has been an active research area, and a comprehensive survey of various methods is given in Prakasa Rao [1].

As fractional Brownian motion plays an important role in the modeling of financial time series, there has been a growing interest in the study of similar problems for stochastic processes driven by fractional Brownian motion $(\mathrm{fBm})$ in view of their applications to long-range dependence of time series. A stationary sequence $\left(X_{n}\right)_{n \in \mathbb{N}}$ exhibits long-range dependence if the autocovariance functions $\rho(n):=\operatorname{cov}\left(X_{k}, X_{k+1}\right)$ satisfy

$$
\lim _{n \rightarrow \infty} \frac{\rho(n)}{c n^{-\alpha}}=1
$$

for some constant $c$ and $\alpha \in(0,1)$. In this case, the dependence between $X_{k}$ and $X_{k+n}$ decays slowly as $n \rightarrow \infty$ and

$$
\sum_{n=1}^{\infty} \rho(n)=\infty
$$

(see, e.g., [2, Definition 1.4.1, p.9]). The long-range dependence was first observed by the hydrologist Hurst [3] on projects involving the design of reservoirs along the Nile river. It was also observed that a similar phenomenon occurs in problems concerning traffic

O2014 Shen and Xu; licensee Springer. This is an Open Access article distributed under the terms of the Creative Commons Attribution License (http://creativecommons.org/licenses/by/2.0), which permits unrestricted use, distribution, and reproduction in any medium, provided the original work is properly cited. 
patterns of packet flows in high-speed data networks such as the Internet $($ see $[4,5])$ and in macroeconomics and finance (see [6]).

The problem of parameter estimation and filtering in a simple linear model driven by a fractional Brownian motion was studied by Le Breton [7] in the continuous case. Prakasa Rao $[8,9]$ studied parametric estimation for more general classes of stochastic processes satisfying the linear stochastic differential equations driven by fractional Brownian motion, observed over a fixed period of time $T$. And Prakasa Rao [10] also studied the conditions for such a phenomenon for estimating the drift parameter of a fractional OrnsteinUhlenbeck type process. For the case of discrete data, the problem of parameter estimation was studied in [11,12]. The paper [13] obtained the LSE for fractional Ornstein-Uhlenbeck processes and derived the asymptotic normality of this LSE by using Malliavin calculus. The problem of estimating the parameters in the discrete case has also been given considerable attention (see, e.g., $[14,15])$.

In case of diffusion type processes driven by fractional Brownian motions, a popular method is the maximum likelihood estimators (MLE). The MLE of the drift parameter has also been extensively studied (see, e.g., [16, 17]). Moreover, in recent years, the papers [1820] studied the asymptotic properties of MLE for the drift parameter in some fractional diffusion systems. However, MLE has some shortcomings, its expression of a likelihood function is not explicitly computable. Moreover, MLE is not robust, which means that the properties of MLE will be changed by a slight perturbation. In order to overcome this difficulty, the minimum distance approach is proposed. For a more comprehensive discussion of the properties of minimum distance estimators, we refer to Millar [21].

Following the work of Kutoyants and Pilibossian [22], Prakasa Rao [23] studied the minimum $L_{1}$-norm estimator $\theta_{\varepsilon}^{*}$ of the drift parameter of a fractional Ornstein-Uhlenbeck type process and proved that $\varepsilon^{-1}\left(\theta_{\varepsilon}^{*}-\theta\right)$ converges in probability under $P_{\theta_{0}}$ to a random variable $\zeta$. However, it is not clear what the distribution of $\zeta$ is, so it would be interesting to study the distribution of $\zeta$. In this paper we will study the asymptotic law of its limit distribution for $T \rightarrow+\infty$.

\section{Preliminaries}

Let $\left(\Omega, \mathcal{F},\left\{\mathcal{F}_{t}\right\}_{t \geq 0}, \mathbb{P}\right)$ be a basic complete filtered probability space satisfying the usual conditions, i.e., the filtration is continuous on the right and $\mathcal{F}_{0}$ contains all $\mathbb{P}$-null sets. We consider the parameter estimation problem for a special fractional process, i.e., fractional Ornstein-Uhlenbeck process, which satisfies the following stochastic differential equation:

$$
d X_{t}=\theta X_{t} d t+\varepsilon d B_{t}^{H}, \quad 0 \leq t \leq T, X_{0}=x_{0},
$$

where the drift parameter $\theta \in \Theta=\left(\theta_{1}, \theta_{2}\right) \subseteq \mathbf{R}$ is unknown, $\varepsilon>0$ and $B^{H}=\left\{B_{t}^{H}(t), 0 \leq t \leq\right.$ $T\}$ is a scalar fractional Brownian motion defined on the probability space $\left(\Omega, \mathcal{F},\left\{\mathcal{F}_{t}\right\}_{t \geq 0}\right.$, $\mathbb{P})$. For a fractional Brownian motion $B^{H}$ with Hurst parameter $H \in\left(\frac{1}{2}, 1\right)$, we mean that it is a continuous and centered Gaussian process with the covariance function

$$
E\left(B_{s}^{H} B_{t}^{H}\right)=\frac{1}{2}\left[s^{2 H}+t^{2 H}-|s-t|^{2 H}\right], \quad t \geq 0, s \geq 0 .
$$

By [2] (see Definitions 1.5.1 and 1.5.2, p.11), we introduce the following. 
Definition 1 We say that an $\mathbb{R}^{d}$-valued random process $X=\left(X_{t}\right)_{t \geq 0}$ is self-similar or satisfies the property of self-similarity if for every $a>0$ there exists $b>0$ such that

$$
\operatorname{Law}\left(X_{a t}, t \geq 0\right)=\operatorname{Law}\left(b X_{t}, t \geq 0\right),
$$

where Law $(\cdot)$ denotes the law of random variable $\cdot$.

Remark 1 Note that (3) means that the two processes $X_{a t}$ and $X_{b t}$ have the same finitedimensional distribution functions, i.e., for every choice $t_{0}, \ldots, t_{n}$ in $\mathbb{R}$,

$$
P\left(X_{a t_{0}} \leq x_{0}, \ldots, X_{a t_{n}} \leq x_{n}\right)=P\left(X_{b t_{0}} \leq x_{0}, \ldots, X_{b t_{n}} \leq x_{n}\right)
$$

for every $x_{0}, \ldots, x_{n}$ in $\mathbb{R}$.

Definition 2 If $b=a^{H}$ in the above definition, then we say that $X=\left(X_{t}\right)_{t \geq 0}$ is a self-similar process with Hurst index $H$ or that it satisfies the property of (statistical) self-similar process with Hurst index $H$. The quantity $D=1 / H$ is called the statistical fractal dimension of $X$.

Remark 2 Note that the law of a Gaussian random variance is determined by its expectation value and variation. By (2), it is easy to see that $B^{H}$ is a self-similar process with Hurst index $H$. Let

$$
B_{T}^{H *}:=\sup _{0 \leq t \leq T} B_{t}^{H}
$$

Then we conclude from the fact that $B^{H}$ is a self-similar process with Hurst index $H$ that

$$
\operatorname{Law}\left(B_{a t}^{H^{*}}\right)=\operatorname{Law}\left(a^{H} B_{t}^{H *}\right), \quad a>0, t \geq 0 .
$$

Let $\theta_{0}$ be the true parameter of $\theta \in \Theta=\left(\theta_{1}, \theta_{2}\right) \subseteq \mathbf{R}$, and $x_{t}(\theta):=x_{0} e^{\theta t}, 0 \leq t \leq T$, the solution of (1) with $\varepsilon=0$. It is easy to see from (1) that

$$
X_{t}-x_{t}\left(\theta_{0}\right)=\varepsilon e^{\theta_{0} t} \int_{0}^{t} e^{-\theta_{0} s} d B_{s}^{H} .
$$

In this paper, we are concerned with the minimum $L_{1}$-norm estimate $\theta_{\varepsilon}^{*}$ defined by

$$
\theta_{\varepsilon}^{*}:=\arg \inf _{\theta \in \Theta} \int_{0}^{T}\left|X_{t}-x_{t}(\theta)\right| d t .
$$

Set

$$
Y_{t}:=e^{\theta_{0} t} \int_{0}^{t} e^{-\theta_{0} s} d B_{s}^{H}
$$

which is a Gaussian process and can be interpreted as the 'derivative' of the process $\left\{X_{t}, 0 \leq\right.$ $t \leq T\}$ with respect to $\varepsilon$. 
Introduce the random variable

$$
\zeta_{T}:=\arg \inf _{-\infty<u<\infty} \int_{0}^{T}\left|Y_{t}-u t x_{0} e^{\theta_{0} t}\right| d t .
$$

Let $\mathbb{P}_{\theta_{0}}$ be the probability measure induced by the process $\left\{X_{t}\right\}$ when $\theta_{0}$ is the true parameter and $\varepsilon \rightarrow 0$. So, hereafter, we denote $\zeta=\zeta_{T}, C$ is a constant.

Theorem 1 As $\varepsilon \rightarrow 0$, the random variable $\varepsilon^{-1}\left(\theta_{\varepsilon}^{*}-\theta_{0}\right)$ converges in probability to a random variable whose probability distribution is the same as that of $\zeta$ under $\mathbb{P}_{\theta_{0}}$.

The above theorem due to Prakasa Rao [23] describes the behavior of $\varepsilon^{-1}\left(\theta_{\varepsilon}^{*}-\theta_{0}\right)$. Though the distribution of $\zeta$ is not clear, we can consider its limiting behavior as $T \rightarrow+\infty$.

\section{Asymptotic law}

Theorem 2 Suppose that $\theta_{1}>0$, let $\delta_{T}=x_{0} T\left(2 \theta_{0}\right)^{1-H} \zeta_{T}$, then as $T \rightarrow \infty$, we have $\operatorname{Law}\left(\delta_{T}\right) \Rightarrow \operatorname{Law}\left(B^{H}(1)\right)$.

Proof Using the properties of the stochastic integral, we can write

$$
Y_{t}=e^{\theta_{0} t} \int_{0}^{t} e^{-\theta_{0} s} d B_{s}^{H}=e^{\theta_{0} t} \tilde{B}^{H}\left(\int_{0}^{t} e^{-2 \theta_{0} s} d s\right) .
$$

By applying Remark 2,

$$
Y_{t}=e^{\theta_{0} t} \tilde{B}^{H}\left(\frac{1-e^{-2 \theta_{0} t}}{2 \theta_{0}}\right)=\frac{e^{\theta_{0} t}}{\left(2 \theta_{0}\right)^{H}} \tilde{B}^{H}\left(1-e^{-2 \theta_{0} t}\right),
$$

where $\tilde{B}^{H}(\cdot)$ is a fractional Brownian motion.

Let

$$
\begin{aligned}
& \gamma=1-e^{-2 \theta_{0} t}, \quad 0 \leq \gamma \leq T^{*}=1-e^{-2 \theta_{0} T}, \\
& \omega=\frac{u x_{0}}{\left(2 \theta_{0}\right)^{H}} \ln \left(\frac{1}{1-T^{*}}\right), \quad u \in \mathbb{R} .
\end{aligned}
$$

After changing the variables $t$ and $u$ in the definition of $\zeta_{T}$, we have

$$
\begin{aligned}
\zeta_{T} & =\arg \inf _{-\infty<u<\infty} \int_{0}^{T^{*}}\left|B^{H}(\gamma)-\frac{u x_{0}}{\left(2 \theta_{0}\right)^{H}} \ln \frac{1}{1-\gamma}\right| \frac{1}{(1-\gamma)^{3 / 2}} d \gamma \\
& =\frac{\left(2 \theta_{0}\right)^{H}}{x_{0} \ln \frac{1}{1-T^{*}}} \arg \inf _{-\infty<\omega<\infty} \int_{0}^{T^{*}}\left|B^{H}(\gamma)-\omega \frac{\ln \frac{1}{1-\gamma}}{\ln \frac{1}{1-T^{*}}}\right| \frac{1}{(1-\gamma)^{3 / 2}} d \gamma=\frac{\left(2 \theta_{0}\right)^{H}}{x_{0} \ln \frac{1}{1-T^{*}}} \delta_{T} .
\end{aligned}
$$

We can see that the distribution of the random variable $\zeta_{T}$ depends on three parameters $\theta_{0}, x_{0}$ and $\gamma$, but after changing there is only one parameter $T^{*}$ that the distribution of the random variable $\delta_{T}$ depends on.

Then, we just need to show that for every $\eta>0$,

$$
\lim _{T \rightarrow \infty} \mathbb{P}_{\theta_{0}}\left\{\left|\delta_{T}-B^{H}(1)\right|>\eta\right\}=0
$$


For this purpose, we define the set

$$
V_{\eta}=\left\{\omega \in R: \omega-B^{H}(1)>\eta\right\} .
$$

Let

$$
J(\omega)=\int_{0}^{T^{*}}\left|B^{H}(\gamma)-\omega \frac{\ln \frac{1}{1-\gamma}}{\ln \frac{1}{1-T^{*}}}\right| \frac{1}{(1-\gamma)^{3 / 2}} d \gamma .
$$

Then

$$
\delta_{T}:=\arg \inf _{-\infty<\omega<\infty} J(\omega) .
$$

We have

$$
\begin{aligned}
J\left(B^{H}(1)\right) & =\int_{0}^{T^{*}}\left|B^{H}(\gamma)-B^{H}(1) \frac{\ln \frac{1}{1-\gamma}}{\ln \frac{1}{1-T^{*}}}\right| \frac{1}{(1-\gamma)^{3 / 2}} d \gamma \\
& \leq \int_{0}^{T^{*}} \frac{\left|B^{H}(\gamma)-B^{H}(1)\right|}{(1-\gamma)^{3 / 2}} d \gamma+\frac{\left|B^{H}(1)\right|}{\ln \frac{1}{1-T^{*}}} \int_{0}^{T^{*}} \frac{\ln \frac{1-\gamma}{1-T^{*}}}{(1-\gamma)^{3 / 2}} d \gamma \\
& =: I_{1}+I_{2} .
\end{aligned}
$$

As $T^{*} \rightarrow 1$, we get

$$
\begin{aligned}
\sqrt{1-T^{*}} \ln \frac{1}{1-T^{*}} \mathbb{E}\left\{\left|I_{1}\right|\right\} & \leq C \sqrt{1-T^{*}} \ln \frac{1}{1-T^{*}} \int_{0}^{T^{*}} \frac{(1-\omega)^{H}}{(1-\omega)^{3 / 2}} d \omega \\
& \leq C(H-1 / 2)^{-1} \sqrt{1-T^{*}} \ln \frac{1}{1-T^{*}}\left[1-\left(1-T^{*}\right)^{(H-1 / 2)}\right] \rightarrow 0
\end{aligned}
$$

and

$$
\begin{aligned}
\sqrt{1-T^{*}} \ln \frac{1}{1-T^{*}} \mathbb{E}\left\{I_{2}\right\} & =\sqrt{1-T^{*}} \ln \frac{1}{1-T^{*}} \frac{\left|B^{H}(1)\right|}{\ln \frac{1}{1-T^{*}}} \frac{1}{\sqrt{1-T^{*}}} \int_{1}^{\frac{1}{1-T^{*}}} \frac{\ln x}{x^{3 / 2}} d x \\
& =\left|B^{H}(1)\right| \int_{1}^{\frac{1}{1-T^{*}}} \frac{\ln x}{x^{3 / 2}} d x<+\infty .
\end{aligned}
$$

So as $T \rightarrow+\infty$ corresponds to $T^{*} \rightarrow 1$,

$$
\lim _{T^{*} \rightarrow 1} J\left(B^{H}(1)\right) \sqrt{1-T^{*}} \ln \frac{1}{1-T^{*}}<\infty \quad \text { in } \mathbb{P}_{\theta_{0}} \text {-probability. }
$$

At the same time, we get, for $\omega \in V_{\eta}$,

$$
\begin{aligned}
J(\omega) & =\int_{0}^{T^{*}}\left|B^{H}(\gamma)-B^{H}(1) \frac{\ln \frac{1}{1-\gamma}}{\ln \frac{1}{1-T^{*}}}+\left(B^{H}(1)-\omega\right) \frac{\ln \frac{1}{1-\gamma}}{\ln \frac{1}{1-T^{*}}}\right| \frac{1}{(1-\gamma)^{3 / 2}} d \gamma \\
& \geq\left|B^{H}(1)-\omega\right| \int_{0}^{T^{*}} \frac{\ln \frac{1}{1-\gamma}}{\ln \frac{1}{1-T^{*}}} \frac{1}{(1-\gamma)^{3 / 2}} d \gamma
\end{aligned}
$$




$$
\begin{aligned}
& -\int_{0}^{T^{*}}\left|B^{H}(\gamma)-B^{H}(1) \frac{\ln \frac{1}{1-\gamma}}{\ln \frac{1}{1-T^{*}}}\right| \frac{1}{(1-\gamma)^{3 / 2}} d \gamma \\
\geq & \eta \int_{0}^{T^{*}} \frac{1}{(1-\gamma)^{3 / 2}} d \gamma-\eta \int_{0}^{T^{*}}\left(1-\frac{\ln \frac{1}{1-\gamma}}{\ln \frac{1}{1-T^{*}}}\right) \frac{1}{(1-\gamma)^{3 / 2}} d \gamma-J\left(B^{H}(1)\right) \\
= & 2 \eta\left(\frac{1}{\sqrt{1-T^{*}}}-1\right)-\frac{\eta}{\ln \frac{1}{1-T^{*}}} \int_{0}^{T^{*}} \ln \frac{1-\gamma}{1-T^{*}} \frac{1}{(1-\gamma)^{3 / 2}} d \gamma-J\left(B^{H}(1)\right) .
\end{aligned}
$$

Then

$$
\begin{aligned}
& \inf _{\omega \in V_{\eta}} J(\omega) \sqrt{1-T^{*}} \ln \frac{1}{1-T^{*}} \\
& \geq 2 \eta\left(1-\sqrt{1-T^{*}}\right) \ln \frac{1}{1-T^{*}}-\eta \int_{0}^{\frac{1}{\left(1-T^{*}\right)}} \frac{\ln x}{x^{3 / 2}} d x-\sqrt{1-T^{*}} \ln \frac{1}{1-T^{*}} J\left(B^{H}(1)\right) \\
& \geq \eta \ln \frac{1}{1-T^{*}} .
\end{aligned}
$$

So as $T \rightarrow \infty$ corresponds to $T^{*} \rightarrow 1$,

$$
\inf _{\omega \in V_{\eta}} J(\omega) \sqrt{1-T^{*}} \ln \frac{1}{1-T^{*}} \rightarrow+\infty \quad \text { in } \mathbb{P}_{\theta_{0}} \text {-probability. }
$$

From (15) and (16), we obtain that as $T \rightarrow+\infty$ corresponds to $T^{*} \rightarrow 1$,

$$
\frac{\inf _{\omega \in V_{\eta}} J(\omega)}{J\left(B^{H}(1)\right)} \rightarrow+\infty \quad \text { in } \mathbb{P}_{\theta_{0}} \text {-probability. }
$$

By (13), we get

$$
\delta_{T}=\inf _{\omega \in V_{\eta}} J(\omega) \leq J\left(B^{H}(1)\right) .
$$

Therefore, from relation (17), for any $\eta>0$, we have the result (12).

In other words,

$$
\operatorname{Law}\left(\delta_{T}\right) \quad \Rightarrow \quad \operatorname{Law}\left(B^{H}(1)\right) .
$$

This completes the proof.

\section{Conclusion}

It is interesting to note that, for $T \rightarrow \infty$, although the distribution of $\zeta$ is not clear, we can obtain the asymptotic law of its limit distribution. Furthermore, they can also be obtained in the case of $L_{2}$-norm and $L_{p}$-norm.

Competing interests

The authors declare that they have no competing interests.

Authors' contributions

All authors contributed equally to the manuscript. All authors read and approved the final manuscript. 


\section{Acknowledgements}

We are very grateful to the anonymous referees and the associate editor for their careful reading and helpful comments.

\section{Received: 30 November 2013 Accepted: 11 February 2014 Published: 25 Feb 2014}

\section{References}

1. Prakasa Rao, BLS: Statistical Inference for Diffusion Type Processes. Oxford University Press, London (1999)

2. Biagini, F, Hu, Y, Bernt, $\varnothing$, Zhang, T: Stochastic Calculus for Fractional Brownian Motion and Applications. Probability and Its Applications. Springer, Berlin (2008)

3. Hurst, HE: Long term storage capacity of reservoirs (with discussion). Trans. Am. Soc. Civ. Eng. 116, 770-808 (1951)

4. Willinger, W, Paxson, V, Riedi, R, Taqqu, MS: Long-range dependence and data network traffic. In: Theory and Applications of Long-Range Dependence, pp. 373-407 (2003)

5. Norros, I, Valkeila, E, Virtamo, J: An elementary approach to a Girsanov type formula and other analytical results on fractional Brownian motion. Bernoulli 5, 571-587 (1999)

6. Henry, M, Zafforoni, P: The long-range dependence paradigm for macroeconomics and finance. In: Theory and Applications of Long-Range Dependence, pp. 417-438 (2003)

7. Le Breton, A: Filtering and parameter estimation in a simple linear model driven by a fractional Brownian motion. Stat. Probab. Lett. 38, 263-274 (1998)

8. Prakasa Rao, BLS: Parametric estimation for linear stochastic differential equations driven by fractional Brownian motion. Random Oper. Stoch. Equ. 11, 229-242 (2003)

9. Prakasa Rao, BLS: Berry-Esseen bound for MLE for linear stochastic differential equations driven by fractional Brownian motion. J. Korean Stat. Soc. 34, 281-295 (2005)

10. Prakasa Rao, BLS: Sequential estimation for fractional Ornstein-Uhlenbeck type process. Seq. Anal. 23, 33-44 (2004)

11. Bertin, K, Torres, S, Tudor, CA: Drift parameter estimation in fractional diffusions driven by perturbed random walks. Stat. Probab. Lett. 81, 243-249 (2011)

12. Hu, Y-Z, Nualart, D, Xiao, W-L, Zhang, W-G: Exact maximum likelihood estimator for drift fractional Brownian motion at discrete observation. Acta Math. Sci. 31, 1851-1859 (2011)

13. Hu, Y, Nualart, D: Parameter estimation for fractional Ornstein-Uhlenbeck processes. Stat. Probab. Lett. 80, 1030-1038 (2010)

14. Taqqu, MS: The modelling of ethernet data and of signals that are heavy-tailed with infinite variance. Scand. J. Stat. 29, 273-295 (2002)

15. Tsai, H, Chan, KS: Temporal aggregation of stationary and non-stationary continuous-time processes. Scand. J. Stat. 32, 583-597 (2005)

16. Kleptsyna, ML, Le Breton, A: Statistical analysis of the fractional Ornstein-Uhlenbeck type process. Stat. Inference Stoch. Process. 5, 229-248 (2002)

17. Tudor, CA, Viens, F: Statistical aspects of the fractional stochastic calculus. Ann. Stat. 35, 1183-1212 (2007)

18. Brouste, A, Kleptsyna, M: Asymptotic properties of MLE for partially observed fractional diffusion system. Stat. Inference Stoch. Process. 13, 1-13 (2010)

19. Brouste, A: Asymptotic properties of MLE for partially observed fractional diffusion system with dependent noises. J. Stat. Plan. Inference 140, 551-558 (2010)

20. Brouste, A, Kleptsyna, M, Popier, A: Design for estimation of the drift parameter in fractional diffusion systems. Stat. Inference Stoch. Process. 15(2), 133-149 (2012)

21. Millar, PW: A general approach to the optimality of the minimum distance estimators. Trans. Am. Math. Soc. 286(1), 377-418 (1984)

22. Kutoyants, Y, Pilibossian, P: On minimum $I_{1}$-norm estimate of the parameter of the Ornstein-Uhlenbeck process. Stat. Probab. Lett. 20(2), 117-123 (1994)

23. Prakasa Rao, BLS: Minimum /1 -norm estimation for fractional Ornstein-Uhlenbeck type process. Theory Probab. Math Stat. 71, 181-189 (2004)

10.1186/1687-1847-2014-75

Cite this article as: Shen and Xu: Asymptotic law of limit distribution for fractional Ornstein-Uhlenbeck process. Advances in Difference Equations 2014, 2014:75

\section{Submit your manuscript to a SpringerOpen ${ }^{\circ}$ journal and benefit from:}

- Convenient online submission

- Rigorous peer review

- Immediate publication on acceptance

Open access: articles freely available online

- High visibility within the field

- Retaining the copyright to your article 